\title{
A Review on Metallic Silver Nanoparticles
}

\author{
Bekkeri swathy \\ Palamuru University
}

\begin{abstract}
Nanotechnology refers to the branch of science and engineering dedicated to materials, having dimensions in the order of 100th of $\mathrm{nm}$ or less. The term being new, but has been widely used for the development of more efficient technology. In recent years, nanotechnology has been embraced by industrial sectors due to its applications in the field of electronic storage systems, biotechnology, magnetic separation and pre concentration of target analytes, targeted drug delivery and vehicles for gene and drug delivery. Consequently, with wide range of applications available, these particles have potential to make a significant impact to the society. In general, nanoparticles used in the field of biotechnology range in particle size between 10 and $500 \mathrm{~nm}$, seldom exceeding $700 \mathrm{~nm}$. The nanosize of these particles allows various communications with biomolecules on the cell surfaces and within the cells in way that can be decoded and designated to various biochemical and physiochemical properties of these cells. Similarly, its potential application in drug delivery system and noninvasive imaging offered various advantages over conventional pharmaceutical agents. More specific targeting systems are designed to recognize the targeted cells such as cancer cells. This can be achieved by conjugating the nanoparticle with an appropriate ligand, which has a specific binding activity with respect to the target cells. In addition, nanoparticles provide a platform to attach multiple copies of therapeutic substance on it and hence increase the concentration of therapeutic and diagnostic substances at the pathological site. Also, the concentration and dynamics of the active molecule can be varied by controlling the particle size of nanoparticles $(>3-5 \mathrm{~nm})$.
\end{abstract}

Keywords: nanotechnology, methods of synthesis, characterization, applications, future aspects

\section{INTRODUCTION}

Nanotechnology plays an increasingly crucial role in many key technologies of the new millennium.

The application of nanoscale materials and structures, usually ranging from 1 to $100 \mathrm{~nm}$, is an emerging area of nanoscience and nanotechnology. Nanomaterials may provide solutions to technological and environmental challenges in the areas of solar energy conversion, catalysis, medicine, and water treatment ${ }^{1}$. Nanomaterials often show unique and considerably changed physical, chemical and biological properties compared to their macroscaled counterparts ${ }^{[2]}$. The noble metals, especially silver and gold, have attracted great attention due to their innumerable applications in various branches of science, namely catalysis, photonics, photography, chemicalsensing, Surface Enhanced Raman Scattering (SERS), and most importantly, in the medicinal field as anti-microbial agents ${ }^{[3]}$ Colloidal silver is of particular interest because of its distinctive properties, such as good conductivity, chemical stability, catalytic and antibacterial activity.Silver nanoparticles have many important applications that include spectrally selective coating for solar energy absorption and intercalation material for electrical batteries, as optical receptors, polarizing filters, catalysts in chemical reaction, biolabelling, and as antimicrobial agents ${ }^{[5]}$. There has been an extraordinary growth in nanoscience and technology in recent years, mainly due to both the development of new techniques to synthesize nanomaterials and the accessibility of tools for the classification and manipulation of nanoparticles ${ }^{[4]}$. Production of nanoparticles requires understanding the fundamentals of nanoscale chemistry and physics, as well as the know-how to commercialize them. Broadly speaking, there are two approaches to nanoparticle production: top-down and bottom-up.The former makes a material decrease its size from large to nanoscale, whereas the latter produces nanomaterials by starting from the atomic level ${ }^{[6]}$. Generally, metal nanoparticles can be prepared and stabilized by chemical, physical and biological methods; the chemical approach, such as chemical reduction, electrochemical techniques, photochemical reduction2 and pyrolysis and physical methods, such as Arc-discharge and physical vapor condensation $(\mathrm{pvc})^{[7]}$ is used. Living organisms have huge potential for the production of nanoparticles/nanodevices of wide applications. However, the elucidation of the exact mechanism of nanoparticles production using living organisms needs much more experimentation ${ }^{[8]}$. Studies have shown that the size, morphology, stability and properties (chemical and physical) of the metal nanoparticles are influenced strongly by the experimental conditions, the kinetics of interaction of metal ions with reducing agents, and adsorption processes of stabilizing agent with metal nanoparticles. Hence, the design of a synthesis method in which the size, morphology, stability and properties are controlled has become a major field of interest. 
II. CHARACTERISTICS OF MNPs:

The main characteristics of MNPs

- Large surface-area-to-volume ratio as compared to the bulk equivalents;

- Large surface energies

- The transition between molecular and metallic states providing specific electronic structure (local density of states ldos);

- Plasmon excitation;

- Quantum confinement;

- Short range ordering;

- Increased number of kinks;

- A large number of low-coordination sites such as corners and edges, having a large number of "danglingbonds" and consequently specific and chemical properties and the ability to store excess electrons.

\section{GOALS AND PROBLEMS IN MNPs:}

Ideally,metallic nanoparticles should be prepared by a method which:is reproducible may control the shape of the particlesyields monodisperse metallic nanoparticles is easy, cheap, use less toxis precursors: in water or more environmentally benign solvents (e.g. ethanol) use the least number of reagents used a reaction temperature close to room temperature with as few synthetic steps as possible (one-pot reaction) minimizing the quantities of generated by-products and waste. Silver nanoparticles exhibits the highest efficiency of Plasmon excitation is becoming an increasingly important material in many technologies is the only material whose Plasmon resonance can be of any wavelength in the visible spectrum.

\section{GENERAL METHODS OF PREPARATION OF SILVER NANOPARTICLES:}

- Synthesis methods

- Traditional methods

- Non traditional methods

- Aqueous solution reduction

- Microemulsiontechniques

- Gammaradiation induced methods

- Photoreduction of Ag Ions

\section{GOALS AND PROBLEMS REGARDING THE SYNTHESIS OF AG NPS}

With the traditional methods, the major problem is often a limited flexibility in the size of particles that can be produced and such methods are usually sold on their ability to make $<10 \mathrm{~nm}$ The major problems for the non-traditional methods are often a wide size distribution, lack of particle crystallinity, and the cost and scalability of the production Synthesizing uniform and stable silver nanoparticleswith a controllable size is difficult . The optimum synthetic method should address all of the above problems and additionally yield particles with no extraneous chemicals that can potentially alter the particle's optical properties and surface chemistry. 


\section{MECHANISM OF ACTION}

Silver and most silver compounds have an oligodynamic effect and are toxic for bacteria, algae, and fungi. Among the elements that have this effect, silver is the least toxic for humans. The antibacterial action of silver is dependent on the silver ion. The effectiveness of silver compounds as an antiseptic is based on the ability of the biologically active silver ion $(\mathrm{Ag}+)$ to irreversibly damage key enzyme systems in the cell membranes of pathogens. The antibacterial action of silver has long been known to be enhanced by the presence of an electric field. Applying an electric current across silver electrodes enhances antibiotic action at the anode, likely due to the release of silver into the bacterial culture. The antibacterial action of electrodes coated with silver nanostructures is greatly improved in the presence of an electric field.

\section{SILVER NANOPARTICLES SYNTHESIS}

\section{CHEMICAL METHODS ${ }^{[2]}$}

Chemical methods are usually used to synthesize silver nanoparticles and among them chemical reduction is the most frequently applied method for the preparation of stable, colloidal dispersions in water or organic solvents.2. However, there is no special boundary between the different chemical methods to synthesize Ag NPs which can be classified into the following methods.

\section{Chemical reduction ${ }^{[9]}$}

Typical reducing agents include polyols, $\mathrm{NaBH} 4, \mathrm{~N} 2 \mathrm{H} 4$, sodium citrate and $\mathrm{N}, \mathrm{N}$-dimethyformamide.9 Doubtless, in order to prevent aggregation ofAg NPs, it needs to stabilize with capping agents such as Sodium dodecyl sulphate (SDS), polyvinyl pyrrolidone (pvp), tri-sodium citrate. Some of the chemical reducing reactions can be carried out at room temperature. However, most of them need elevated temperatures for a higher reaction rate ${ }^{[11]}$. Thermal methods such as reduction of $\mathrm{Ag}+$ by dextrose $\mathrm{e}^{[10]}$ and/or hydrazine ${ }^{[11]}$ as a reduction agent and the well-known Tollen's reduction with reducing agent of m-hydroxy banzaldehide are from chemical reduction methods. Nanoparticle morphologies strongly depend on the temperature adopted during the synthesis. To develop a flower-like silver nano architecture at room temperature with size $20 \mathrm{~nm}$, ascorbic acid was used as the reducing agent while citric acid ${ }^{[13,14]}$ was found to play a key role in the nanostructure formation. Silver nanoparticles of $40-80 \mathrm{~nm}$ size are formed in the process of oxidation of glucose to gluconic acid by amine in the presence of silver nitrate, and the gluconic acid caps the nanosilver particle. Silver nanoparticles have been synthesized by the polyol process with the assistance of supercritical carbon dioxide (SCCO2), with silver nitrate used as the base material, polyvinyl pyrrolidone (PVP) as the stabilizer for the silver clusters, and ethylene glycol as the reducing agent and solvent. Polyvinyl pyrrolidone not only protected the nanosize silver particles from aggregation, but it also promoted nucleation. The silver nanoparticles synthesized by $\mathrm{SCCO} 2$ were smaller and had a more even dispersion than those made under the same conditions by the conventional heating process ${ }^{[16]}$. The resultant nanosilver colloid ${ }^{[17,18]}$ had low toxicity and high stabilization, and the particles were all spherical with mean diameters in the range of $2-5 \mathrm{~nm}^{[21]}$.

\section{Photochemical method ${ }^{[23]}$}

$\mathrm{Ag}$ NPs can be successfully synthesized by using a variety of irradiation methods. For example, laser irradiation of an aqueous solution of Ag salt and surfactant can fabricate Ag NPs of well defined shape and size distribution. No reducing agent is required.Silver nanoparticles having narrow size distribution were synthesized in ethylene glycol-water mixtures without the use of a stabilizer. They used pulse radiolysis method to produce nanoparticles by silver perchlorate. Reduction of silver ions was achieved using UV light instead of chemical materials. Silver nanoparticles were formed in a natural rubber matrix via photo reduction of film cast from natural rubber latex (NRL) containing silver salt; their size ranged 4 to $10 \mathrm{~nm}$.Synthesis procedures using microwave irradiation have also been employed, and this method is known to have a faster heating rate than conventional heating through conduction and convection.

\section{Electrochemical method (electrolysis) ${ }^{[23]}$}

The electrolysis process has long been used for the reduction of metal ions. However, there are a few reports about using this method in the synthesis of metal nanoparticles, especially silver, however this could be classified in the synthesis of Ag NPs. silver nanospheres of average size in the range of $\sim 11 \mathrm{~nm}$ were grown at room temperature by reducing silver nitrate in polyol solution using the electrochemical method in the presence of PVP and KNO3. A rotating disk Ti electrode (6 mm diameter) was used as the cathode, and a $2 \mathrm{~cm}$ diameter Pt plate was used as the anode, which resulted in the formation of electro-deposited $\mathrm{Ag}^{\circ}$ nanoparticles. 
Pyrolysis $^{[24]}$

Another method of synthesizing Ag nanoparticles is spray pyrolysis. nanosilver powder with about $100 \mathrm{~nm}$ average grain size had been fabricated by spray pyrolysis, using AgNO3 solution, $336 \mathrm{~mL} \mathrm{~h}-1$ flux of AgNO3 solution, 0.32 MPa flux of carrier gas and at $720{ }^{\circ} \mathrm{C}$ furnace set temperature.Chemical methods for metal nanoparticle fabrication usually involve toxic chemicals, which can be harmful to our environment. Although these methods may successfully produce pure silver nanoparticles, they require the use of stabilizers to protect the Ag nanoparticles against agglomeration. Additionally, these methods are usually expensive and potentially harmful to the environment. ${ }^{[2]}$

\section{PHYSICAL METHODS ${ }^{[24]}$}

Physical methods do not involve toxic chemicals and they are usually fast. Physical methods include physical vapor condensation (pvc) and Arc-discharge ${ }^{[27]}$.

\section{Physical vapor condensation $(P V C)^{[27]}$}

In order to fabricate nanoparticles, the vaporization method has been frequently used, in which the target materials are vaporized by heat source and then rapidly condensed. The vaporization process can be subdivided into physical and chemical methods depending on whether the reaction is present.

\section{Arc-discharge method ${ }^{[27]}$}

A novel technique for preparing a nanosilver water suspension without surfactants and stabilizers was studied using the arc-discharge method. Silver wires (99.99\%) $1 \mathrm{~mm}$ in diameter submerged in deionized water were used as electrodes. The DC arc-discharge system consists of five main parts: i) two silver electrodes $1 \mathrm{~mm}$ in diameter, ii) a servo control system that maintains a constant distance between the electrodes, iii) a power supply system that controls the DC arc-discharge parameters, iv) a glass container with an electrode holder and deionized water to collect the silver colloids, v) a stirring system with magnetic stirrer and stirring bar.

In order to ionize the aqua medium between the electrodes, the DC arc-discharge system provides a pulse voltage of $70-100 \mathrm{~V}$ for 2-3 ms and then maintains a pulse of $20-40 \mathrm{~V}$ for around 10 _s. During the arcdischarge, the surface layer of the Ag wires evaporates and condenses in the water. The transparent solution converts to a characteristic pale yellow color and then a silver suspension is created.

\section{BIOLOGICAL METHODS ${ }^{[28]}$}

Living organisms such as bacteria, fungi and plants have huge potential for the production of metal nanoparticles. Microorganisms have recently been explored as potential biofactories for the synthesis of metallic nanoparticles such as $\mathrm{CdS}^{[28]}$, Ti/ $/ \mathrm{Ni}^{[29]}$, titanate ${ }^{[30]}$, zirconia ${ }^{[31]}$, gold ${ }^{[32,33]}$ and silver ${ }^{[34,36]}$. The use of microorganisms in the synthesis of nanoparticles emerges as an eco-friendly and exciting approach. On the other hand, researchers have turned to biological synthesis because of the good control over size distribution of nanoparticles.Here, we summarize some of the organisms used in the biosynthesis of Ag nanomaterials and describe the properties that should be inherent for the production of Ag nanoparticles of desired characteristics.

\section{Use of bacteria ${ }^{[37]}$}

To synthesize Ag nanoparticles with size less than $200 \mathrm{~nm}$. Bacteria were grown on Lennox L (LB) agar substrate, containing $50 \mathrm{mmol} \mathrm{L}-1 \mathrm{AgNO} 3$, at $30{ }^{\circ} \mathrm{C}$ for $48 \mathrm{~h}$ in the dark. Biosynthesis of silver nanocrystals by Bacillus licheniformis was studied. Aqueous silver ions were reduced to silver nanoparticles when added to the biomass of B.licheniformis. This was indicated by the change in color from whitish-yellow to brown.The probable mechanism for the formation of silver nanoparticles involves the enzyme nitrate reductase.

\section{APPLICATIONS OF Ag NPS}

\section{Catalysis}

-the selective oxidation of alcohols, alkanes and alkenes

-for the synthesis of industrially interesting products including water splitting degradation of organic pollutants.

2. Biology and Medicine:Ag is a highly antimicrobial material used in:

$$
\text { -water purification }
$$

-wound care

-medical devices

- drog delivery

3. Optics: Optoelectronic devices-active waveguides in optical devices (amplifiers)

4. Electronics : electronically conductive adhesives (ECAs) 


\section{RECENT ADVANCES IN SILVER NANOPARTICLES ${ }^{[38]}$}

Colloidal silver (a colloid consisting of silver particles suspended in liquid) and formulations containing silver salts were used by physicians. Colloidal silver has again been marketed as an alternative medicine, often with extensive "cure-all" claims. Colloidal silver products remain available in many countries as dietary supplements and homeopathic remedies, although they are not effective in treating any known condition and carry the risk of both permanent cosmetic side effects such as argyria and more serious ones such as allergic reactions, and interactions with prescription medications.

The incorporation into wound dressings, creams, and as an antibiotic coating on medical devices. While wound dressings containing silver sulfadiazine or silver nanomaterials may be used on external infections, there is little evidence to support such use. There is tentative evidence that silver coatings on urinary catheters and endotracheal breathing tubes may reduce the incidence of catheter-related urinary tract infections and ventilator-associated pneumonia, respectively The silver ion $(\mathrm{Ag}+)$ is bioactive and in sufficient concentration readily kills bacteria in vitro. Silver exhibits low toxicity in the human body, and minimal risk is expected due to clinical exposure by inhalation, ingestion, dermal application. Silver and silver nanoparticles are used as an antimicrobial in a variety of industrial, healthcare and domestic applications.

\section{Antibacterial cream}

A 2012 systematic review reported that topical silver showed significantly worse healing time compared to controls and showed no evidence of effectiveness in preventing wounds infection. A 2010 Cochrane systematic review concluded that "There is insufficient evidence to establish whether silvercontaining dressings or topical agents promote wound healing or prevent wound infection".

The US Food and Drug Administration has approved a number of topical preparations of silver sulfadiazine for treatment of second- and third-degree burns.

\section{Dressings}

A 2012 systematic review found that silver-containing dressings were no better than non-silvercontaining dressings in treating burns A 2012 Cochrane review found that silver-containing hydrocolloid dressings were no better than standard alginate dressings in treating diabetic foot ulcers. Silver-containing foam resulted in a greater reduction in wound size and more effective control of leakage and odor than non-silver dressings.

\section{Endotracheal tubes}

Limited evidence suggests that endotracheal breathing tubes coated with silver may reduce the incidence of ventilator associated pneumonia (VAP) and delay its onset, although no benefit is seen in the duration of intubation, the duration of stay in intensive care or the mortality rate.

\section{Urinary catheters}

Tentative evidence supports a decreased risk of urinary tract infections when silver-alloy catheters are used.

\section{Other uses}

Silver compounds are used in external preparations as antiseptics, including both silver nitrate and silver proteinate, which can be used in dilute solution as eye drops to prevent conjunctivitis in newborn babies. Silver nitrate is also sometimes used in dermatology in solid stick form as a caustic ("lunar caustic") to treat certain skin conditions, such as corns and warts. Silver is also used in bone prostheses, reconstructive orthopaedic surgery and cardiac devices. Chlorhexidine-silver-sulfadiazine central venous catheters significantly reduce the incidence of catheter-related bloodstream infections (CR-BSI). Silver diamine fluoride is an effective intervention to reduce dental caries (tooth decay).Silver acetate has been used as a potential aid to help stop smoking.

\section{Adverse effects ${ }^{[39]}$}

In animals and humans, silver accumulates in the body. Chronic intake of silver products can result in an accumulation of silver or silver sulphide particles in the skin. These particles in the skin darken with exposure to sunlight, resulting in a blue or gray discoloration of the skin known as argyria Localized argyria can occur as a result of topical use of silver-containing solutions, while generalized argyria results from the ingestion of such substance. 
While argyria is usually limited to skin discoloration, there are isolated reports of more serious neurologic, renal, or hepatic complications caused by ingesting colloidal silver.Colloidal silver may interact with some prescription medications, reducing the absorption of some antibiotics and thyroxine among others.Some people are allergic to silver, and the use of treatments and medical devices containing silver is contraindicated for such people. Water purifications Electrolytically-dissolved silver has been used as water disinfecting agent hospitals filter hot water through copper-silver filters to defeat MRSA and legionella infections. In developing countries for water disinfection (the silver inhibits microbial growth on the filter substrate, to prevent clogging, and does not directly disinfect the filtered water).

\section{CONCLUSION}

Ag NPs are the most important NPs because of their applications. These nanoparticles have many important applications that include spectrally selective coating for solar energy absorption and intercalation material for electrical batteries, as optical receptors, polarizing filters, catalysts in chemical reaction, biolabelling and as antimicrobial agents.Application of silver nanoparticles in these fields is dependent on the ability to synthesize particles with different chemical composition, shape, size, and monodispersity.Generally, there are various methods to synthesize Ag NPs. Many methods are based on the reduction of Ag+ ions in a water solution. These methods use a reducing agent for the reduction of $\mathrm{Ag}+$ ions in solution (especially). In chemical reduction methods, the reducing agent is a chemical solution such as polyols, $\mathrm{NaBH} 4, \mathrm{~N} 2 \mathrm{H} 4$, sodium citrate, and N, N-dimethyformamide, whereas in biological methods, collection of enzymes especially nitrate reductase play such role. Spray pyrolysis methods are carried out in operating conditions of high temperature and pressure; electrochemical methods are based on electrolysis of solution. In the physical method of Arcdischarge, the $\mathrm{Ag}$ metal evaporates in the pure water by electric voltage, then it condenses and produces $\mathrm{Ag}$ NPs. From among the mentioned methods, chemical methods have mostly been applied to synthesize Ag NPs. However, nowadays, this method is used to synthesize Ag NPs in large scales. In some chemical methods, a stabilizer (surfactant) is added to the first solution to prevent agglomeration of Ag NPs, whereas in biological methods there is no need to add a stabilizing agent. Toxicity is a disadvantage of the chemical methods. In addition, many of these methods are energy-intensive, althoughAg NPs are synthesized fast. In contrast, biological methods, as an alternative, are carried out in environmental conditions and consume no energy. Of course, the time required to synthesize Ag NPs is longer compared to chemical methods, although the synthesis time has recently decreased with finding suitable microorganisms or organisms. In addition, the current interest in nanomaterials is focused on the controllable properties of size and shape because the optical, electronic, magnetic, and catalytic properties of metal nanoparticles strongly depend on their sizes and shapes. Controllability in biological methods is far easier to achieve than with other methods. Therefore, the use of microorganisms in the synthesis of nanoparticles emerges as an eco-friendly and exciting approach. In addition, the use of bacteria as a n novel biotechnology to facilitate the production of nanoparticles is in its infancy.

\section{REFERENCES}

[1]. Mandal, D., Bolander, M. E., Mukhopadhyay, D., Sarkar,G., Mukherjee, P., Appl. Microbiol. Biotechnol. 69 (2006) 485 .

[2]. Sharma, V. K., Yngard, R. A., Lin, Y., Adv. Colloid Interfac. 145 (2009) 83.

[3]. Sarkar, S., Jana, A. D., Samanta, S. K., Mostafa, G., Polyhedron 26 (2007) 4419.

[4]. Gardea-Torresdey, J. L., Gomez, E., Peralta-Videa, J. R., Parsons, G. J., Troiani, H., Jose-Yacama, M., Langmuir 19 (2003) 1357

[5]. Bhainsa, K. C., D’Souza, S. F., Colloid Surface B 47 (2006) 160

[6]. Charinpanitkul, T., Faungnawakij, K., Tanthapanichakoon, W., Adv. Powder Technol. 19 (2008) 443.

[7]. Tavakoli, A., Sohrabi, M., Kargari, A., Chem. Pap. 61 (2007) 151

[8]. Mohanpuria, P., Rana, N. K., Yadav, S. K., J. Nanopart. Res. 10 ( 2008) 507.

[9]. Jiang, H., Moon, K. S., Zhang, Z., Pothukuchi, S., Wong, C. P., J. Nanopart. Res. 8 (2006) 117.

[10]. Lu, Y., Chou, K., J. Chin Inst. Chem. Eng. 39 (2008) 673.

[11]. Torres-Cisneros, M., Velásquez-Ordónez, C., Sánchez-Mondragón, J., Campero, A., Ibarra-Manzano, O. G., May-Arrioja, D. A., Plascencia-Mora, H., Espinoza-Calderón, A., Sukhoivanov, I., Microelectr. J. 40 (2009) 618.

[12]. Khanna, P. K., Singh, N., Kulkarni, D., Deshmukh, S., Charan, S., Adhyapak, P. V., Mater. Lett. 61 (2007) 3366.

[13]. Reddy, A. S., Chen, C. Y., Baker, S. C., Chen, C. C., Jean, J. S., Fan, C. W., Chen, H. R., Wang, J. C., Mater. Lett. 63 (2009) 1227.

[14]. Schabes-Retchkiman, P. S., Canizal, G., Herrera-Becerra, R., Zorrilla, C., Liu, H. B., J. A. Opt. Mater. 29 (2006) 95.

[15]. Janardhanan, R., Karuppaiah, M., Hebalkar, N., Rao, T. N., Polyhedron 28 (2009) 2522.

[16]. Chih, Y. W., Cheng, W. T., Mater. Sci. Eng. B 145 (2007) 67.

[17]. Zhang, W., Qiao, X., Chen, J., Colloid Surface A 299 (2007) 22.

[18]. Zhang, W., Qiao, X., Chen, J., Chem. Phys. 330 (2006) 495.

[19]. Akaighe, N., Maccuspie, R. I., Navarro, D. A., Aga, D. S., Banerjee, S., Sohn, M., Sharma, V. K., Environ. Sci. Technol. 45 (2011) 3895.

[20]. Jacob, J. A., Kapoor, S., Biswas, N., Mukherjee, T., Colloid Surface A 301 (2007) 329.

[21]. Remita, S., Fontaine, P., Lacaze, E., Borensztein, Y., Sellame, H., Farha, R., Rochas, C., Goldmann, M., Nucl. 
Instrum. Meth. B 263 (2007) 436.

[22]. Abu Bakar, N. H. H., Ismail, J., Abu Bakar, M., Mater. Chem. Phys. 104 (2007) 276.

[23]. Lim, P. Y., Liu, R. S., She, P. L., Hung, C. F., Shih, H. C., Chem. Phys. Lett. 420 (2006) 304.

[24]. Qiaoxin, Z., Hao, L., Xiaohui, W., Xiaoliang, S., Xinglong, D., J. Wuhan Univ. Technol. 24 (2009) 871

[25]. Shia, X., Wang, S., Duan, X., Zhang, Q., Mater. Chem. Phys. 112 (2008) 1110.

[26]. Sawai, O., Oshima, Y., J. Supercrit. Fluid 47 (2008) 240.

[27]. Tien, D. C., Liao, C. Y., Huang, J. C., Tseng, K. H., Lung, J. K., Tsung, T. T., Kao, W. S., Tsai, T. H., Cheng, T. W., Yu, B. S., Lin, H. M., Stobinski, L., Rev. Adv. Mater. Sci. 18 (2008) 750.

[28]. Kowshik, M., Deshmukh, N., Vogel, W., Urban, J., Kulkarni, S. K., Paknikar, M. K., Biotechnol. Bioeng. 28 (2002) 583.

[29]. Mukherjee, P., Ahmad, A., Mandal, D., Senapati, S., Sainkar, S. R., Khan, M. L., Ramani, R., Parischa, R., Ajayakumar, P. V., Alam, M., Sastry, M., Kumar, R., Angew. Chem. Int. Ed. 40 (2001) 3585.

[30]. Bansal, V., Poddar, P., Ahmad, A., Sastry, M., J. Am. Chem. Soc. 128 (2006) 11958.

[31]. Bansal, V., Rautaray, D., Ahmad, A., Sastry, M., J. Mater. Chem. 14 (2004) 3303.

[32]. Ankamwar, B., Chaudhary, M., Sastry, M., Syn. React. Inorg. Met. Nano 35 (2005) 26.

[33]. Armendariz, V., Herrera, I., Peralta-Videa, J. R., Jose-Yacaman, M., Troiani, H., Santiago, P., Gardea-Torresdey, J.

R., J. Nanopart. Res. 6 (2004) 377.

[34]. Gericke, M., Pinches, A., Hydrometallurgy 83 (2006) 132.

[35]. Roh, Y., Lauf, R. L., McMillan, A. D., Zhang, C., Rawn, C. J., Bai, J., Phelps, J. L., Solid State Commun. 8 (2001) 529 .

[36]. Sastry, M., Ahmad, A., Islam Khan, M., Kumar, R., Curr. Sci. India 85 (2003) 162.

[37]. Panyala, NR, Pena-Mendez, EM, Havel, J: Silver or silver nanoparticles: a hazardous threat to the environment and human health?. J. Appl. Biomed.. 6, 117-129 (2008)

[38]. Allsopp, M, Walters, A, Santillo, D: Nanotechnologies and Nanomaterials in Electrical and Electronic Goods: A Review of Uses and Health Concerns, Greenpeace Research Laboratories, London (2007)

[39]. Hussain, SM, Hess, KL, Gearhart, JM, Geiss, KT, Schlager, JJ: In vitro toxicity of nanoparticles in BRL 3A rat liver cells. Toxicol. In Vitro. 19, 975-983 (20s05).PubMed Abstract | Publisher Full Text 